EPJ Web of Conferences 69, 00017 (2014)

DOI: $10.1051 /$ epjconf / 20146900017

(C) Owned by the authors, published by EDP Sciences, 2014

\title{
Photon and proton induced fission on heavy nuclei at intermedi- ate energies
}

\author{
E. Andrade-II ${ }^{1}$, G. S. Karapetyan ${ }^{1}$, A. Deppman ${ }^{1}$, A. R. Balabekyan², N. A. Demekhina ${ }^{3}$, and V. \\ Guimarães ${ }^{1}$ \\ ${ }^{1}$ Instituto de Fisica, Universidade de São Paulo, P. O. Box 66318, 05389-970 São Paulo, SP, Brazil \\ ${ }^{2}$ Yerevan State University, Alex Manoogian 1, Yerevan 0025, Armenia \\ ${ }^{3}$ Yerevan Physics Institute, Alikhanyan Brothers 2, Yerevan 0036, Armenia Joint Institute for Nuclear Re- \\ search (JINR), Flerov Laboratory of Nuclear Reactions (LNR), Joliot-Curie 6, Dubna 141980, Moscow, Rus- \\ sia
}

\begin{abstract}
We present an analysis of fission induced by intermediate energy protons or photons on actinides. The $660 \mathrm{MeV}$ proton induced reactions are on ${ }^{241} \mathrm{Am},{ }^{238} \mathrm{U}$, and ${ }^{237} \mathrm{~Np}$ targets and the Bremmstrahlung-photons with end-point energies at $50 \mathrm{MeV}$ and $3500 \mathrm{MeV}$ are on ${ }^{232} \mathrm{Th}$ and ${ }^{238} \mathrm{U}$ targets. The study was performed by means of the Monte Carlo simulation code CRISP. A multimodal fission extension was added to the code within an approach which accounts for the contribution of symmetric and asymmetric fission. This procedure allowed the investigation of fission cross sections, fissility, number of evaporated nucleons and fission-fragment charge distributions. The comparison with experimental data show a good agreement between calculations and experiments.
\end{abstract}

\section{Introduction}

For 7 decades now, high energy proton induced reactions have been studied with great interest due to the possibility of investigating fundamental issues such as the nucleon-nucleon interaction and properties of excited nuclei. A large variety of phenomena arise from modifications of proton energy or of the target so that there is still to much to be discovered and understood experimentally and theoretically. In addition, better understanding of proton induced reactions leads usually to more predictive power and control, factors that provide great benefit to applications that are already natural recipients for this knowledge such as nuclear reactor techonlogies and nuclear medicine. When it comes to fission, comparison between calculations and experiments, especially distributions of mass and charge can provide important insights about fragment formation.

Photons can also be a convenient tool to investigate the properties of fissile systems by means of photofission reactions. It is possible not only to study the transformation of the fissioning nucleus at large deformations but the very mechanism of photoabsorption itself as well [1-3] validating, therefore, the accepted models for photon interaction with the nucleus.

The present work shows the results of calculations using the CRISP code with multimodal model in describing proton-induced fission at $660 \mathrm{MeV}$ on ${ }^{241} \mathrm{Am},{ }^{238} \mathrm{U},{ }^{237} \mathrm{~Np}$ and photon-induced fission from Bremmstrahlung at $50 \mathrm{MeV}$ and $3500 \mathrm{MeV}$ end-point energies on ${ }^{238} \mathrm{U}$ and ${ }^{232} \mathrm{Th}$. The data considered here are from the experiments of Refs. [4-7]. 


\section{Methodology}

In this work we use the Monte Carlo simulation code CRISP to calculate nuclear processes triggered by the inelastic interaction of protons with heavy target nuclei. This code has been developed for more than ten years [8-14] and it has been applied in the study of fission induced by photons and protons, and for the study of hyper-nuclear decay [15]. It has also been used in the development of new nuclear reactor technologies [16-18]. The main feature of this Monte Carlo code is the precise description of the intranuclear cascade, where a time-ordered sequence of collisions is governed by strict verification of the Pauli principle in a square-well nuclear model. In this case, pre-equilibrium emissions are naturally considered until the complete thermalization of the nucleus. After the intranuclear cascade is finished, the competition between evaporation and fission is described by using the WeisskopfEwing model [19], until the nucleus is too cold to emit any other particle.

In each step of the evaporation chain, the nuclear excitation energy is recalculated by

$$
E_{f}=E_{i}-(B+V+\epsilon)
$$

where $E_{f}$ and $E_{i}$ are the final and initial excitation energy of the nucleus, $B$ is the separation energy of the evaporated particle, $V$ is Coulomb potencial and $\epsilon$ is the kinetic energy of the emitted particle, fixed at $2 \mathrm{MeV}$ in this work. Whenever fission occurs, the fragments are determined according to the relative intensities of each fission mode, namely Superlong (SL), Standard I and Standard II, respectively, one symmetric and two asymmetric channels. The Random Neck Rupture Model (MM-NRM) [20] has successfully described the fission process by taking into account the collective effects of nuclear deformation during fission with the liquid-drop model, and single-particle effects through microscopic shell-model corrections. The microscopic corrections create valleys in the space of elongation and mass number, each valley corresponding to one different fission mode. The yield of a fragment, characterized by the fragment mass number $\mathrm{A}$ and the atomic number $\mathrm{Z}$, is determined for each mode by a Gaussian distribution.

In the multimodal model, the fission cross section, as a function of mass number, is obtained by the sum of the three Gaussian functions corresponding to the three modes:

$$
\begin{aligned}
\sigma_{A}= & \frac{1}{\sqrt{2 \pi}}\left[\frac{K_{1 A S}}{\sigma_{1 A S}} \exp \left(-\frac{\left(A-A_{S}-D_{1 A S}\right)^{2}}{2 \sigma_{1 A S}^{2}}\right)+\frac{K_{1 A S}}{\sigma_{1 A S}} \exp \left(-\frac{\left(A-A_{S}+D_{1 A S}\right)^{2}}{2 \sigma_{1 A S}^{2}}\right)+\right. \\
& \frac{K_{2 A S}}{\sigma_{2 A S}} \exp \left(-\frac{\left(A-A_{S}-D_{2 A S}\right)^{2}}{2 \sigma_{2 A S}^{2}}\right)+\frac{K_{2 A S}}{\sigma_{2 A S}} \exp \left(-\frac{\left(A-A_{S}+D_{2 A S}\right)^{2}}{2 \sigma_{2 A S}^{2}}\right)+ \\
& \left.\frac{K_{S}}{\sigma_{S}} \exp \left(-\frac{\left(A-A_{S}\right)^{2}}{2 \sigma_{S}^{2}}\right)\right]
\end{aligned}
$$

where $A_{S}$ is the mean mass number determining the center of Gaussian functions; and $K_{i}, \sigma_{i}$, and $\mathrm{D}_{i}$ are the contribution, dispersion and position parameters of the $i^{\text {th }}$ Gaussian functions. The indices $A S$, $S$ designate the asymmetric and symmetric components.

The CRISP code works on an event-by-event basis, and therefore the parameter $A_{S}$ in Eq. (2) is completely determined by the mass of the fissioning nucleus $A_{f}$, that is, $A_{S}=A_{f} / 2$. The quantities $A_{S}$ $+\mathrm{D}_{i A S}=\mathrm{A}_{H}$ and $A_{S}-\mathrm{D}_{i A S}=\mathrm{A}_{L}$, where $A_{H}$ and $A_{L}$ are the masses of the heavy and light fragments, respectively, determine the positions of the heavy and light peaks of the asymmetric components of the mass distribution. The values of $\mathrm{A}_{H}+\mathrm{A}_{L}=2 A_{S}$ are treated as the masses of nuclei that undergo fission in the respective channel. 


\section{$\mathrm{CNR} * 13$}

It is assumed that the charge distribution of the fission fragments is well described by a Gaussian function characterized by the most probable charge, $Z_{p}$, and the associate width parameter, $\Gamma_{z}$, of the distribution [21, 22]:

$$
\sigma_{A, Z}=\frac{\sigma_{A}}{\Gamma_{z} \pi^{1 / 2}} \exp \left(-\frac{\left(Z-Z_{p}\right)^{2}}{\Gamma_{z}^{2}}\right),
$$

where $\sigma_{A, Z}$ is the independent cross section of the nuclide, $Z, A$.

The most probable charge and the corresponding dispersion are simple functions of the mass of the fragment following:

$$
Z_{p}=\mu_{1}+\mu_{2} A
$$

and

$$
\Gamma_{z}=\gamma_{1}+\gamma_{2} A
$$

All fragments obtained go into a final evaporation step according to the model of evaporation/fission competition already mentioned. The energy of each fragment is mass weighted using

$$
E_{i}=\frac{A_{i}}{A_{f}} E_{\text {frag }}
$$

where $E_{i}$ and $A_{i}$ are the excitation energy and the mass number of the fragment $i$, respectively. $E_{\text {frag }}$ is the total excitation energy of the fragments, which is assumed to be equal to the excitation energy of the fissioning system.

\section{Results and Discussion}

The parameters in Eq. (2), (4) and (5) for all targets and reactions studied are presented in Table 1.

The mass distributions of fragments for all proton-induced reactions are shown in Figure 1. It is possible to observe a remarkable agreement with experiment regarding the dispersion and the position of the distributions. Both symmetric and asymmetric contributions can be identified as well positioned, particularly for ${ }^{238} \mathrm{U}$ and ${ }^{237} \mathrm{~Np}$.

However, the underestimation of the experimental cross sections, albeit not very large, is significant and may arise from the fact that the CRISP code calculates the total fission cross section by supposing that it is given by $\sigma_{F}^{\text {calc }}=D \sigma_{\text {in }}$, where $D$ is the nuclear fissility and $\sigma_{\text {in }}$ is the total cross section for the inelastic interaction. The CRISP code adopts the geometric cross section to estimate the inelastic cross sections:

$$
\sigma_{i n} \sim \sigma_{g}=\pi\left(r_{0}+r_{0} A^{1 / 3}\right)^{2}
$$

This geometric cross section considers the nucleus as a sphere of radius $R(A)=r_{0} A^{1 / 3}$ and the proton as a sphere of radius $r_{0}$.

Considering that these are heavy nuclei for which sphericity does not hold, this suposition may contribute to the decrease of the total cross section. The ratios between calculated and experimental total fission cross sections, $\sigma_{F}^{\text {calc }} / \sigma_{F}^{\text {exp }}$ are $0.6 \pm 0.1,0.8 \pm 0.1$ and $0.7 \pm 0.1$, respectiveley for ${ }^{241} \mathrm{Am}$, ${ }^{238} \mathrm{U}$ and ${ }^{237} \mathrm{~Np}$.

In addition, it is possible to observe that the asymmetric contribution in the case of ${ }^{238} \mathrm{U}$ is higher than for the others although they are not very different targets. The reason for this arises from the 
EPJ Web of Conferences

Table 1. Values for parameters in Eq. (2) (4) and (5).

\begin{tabular}{|c|c|c|c|}
\hline & \multicolumn{3}{|c|}{ Proton-induced } \\
\hline Parameter & ${ }^{241} \mathrm{Am}$ & ${ }^{238} \mathrm{U}$ & ${ }^{237} \mathrm{~Np}$ \\
\hline$K_{1 A S}$ & 45.0 & 53.80 & 49.0 \\
\hline$\sigma_{1 A S}$ & 4.2 & 1.60 & 4.5 \\
\hline$D_{1 A S}$ & 20.0 & 22.50 & 21.3 \\
\hline$K_{2 A S}$ & 220.5 & 477.32 & 252.0 \\
\hline$\sigma_{2 A S}$ & 7.0 & 4.29 & 6.5 \\
\hline$D_{2 A S}$ & 25.5 & 22.90 & 26.3 \\
\hline$K_{S}$ & 2970.0 & 1396.45 & 2590.0 \\
\hline$\sigma_{S}$ & 15.0 & 14.2 & 13.7 \\
\hline$\mu_{1}$ & 4.1 & 4.1 & 5.0 \\
\hline$\mu_{2}$ & 0.38 & 0.38 & 0.37 \\
\hline$\gamma_{1}$ & 0.92 & 0.92 & 0.59 \\
\hline \multirow[t]{2}{*}{$\gamma_{2}$} & 0.003 & 0.003 & 0.005 \\
\hline & \multicolumn{3}{|c|}{ Photon-induced (Bremsstrahlung) } \\
\hline Parameter & ${ }^{238} \mathrm{U}-50$ and $3500 \mathrm{MeV}$ & ${ }^{232} \mathrm{Th}-50 \mathrm{MeV}$ & ${ }^{232} \mathrm{Th}-3500 \mathrm{MeV}$ \\
\hline$K_{1 A S}$ & 7.5 & 8.79 & 11.5 \\
\hline$\sigma_{1 A S}$ & 3.54 & 7.07 & 7.3 \\
\hline$D_{1 A S}$ & 15.82 & 17.62 & 12.614 \\
\hline$K_{2 A S}$ & 130.0 & 28.0 & 25.0 \\
\hline$\sigma_{2 A S}$ & 6.0 & 9.5 & 11.5 \\
\hline$D_{2 A S}$ & 20.0 & 21.98 & 20.278 \\
\hline$K_{S}$ & 23.75 & 7.37 & 7.1319 \\
\hline$\sigma_{S}$ & 12.0 & 19.74 & 21.24 \\
\hline$\mu_{1}$ & 4.1 & 5.0 & 5.0 \\
\hline$\mu_{2}$ & 0.38 & 0.37 & 0.37 \\
\hline$\gamma_{1}$ & 0.92 & 0.59 & 0.59 \\
\hline$\gamma_{2}$ & 0.003 & 0.005 & 0.005 \\
\hline
\end{tabular}

fact that there is a critical fissility parameter above which the symmetric contribution begins to prevail and must increase the higher the fissility parameter [23]. In the expression for the critical fissility parameter [23]

$$
\left(Z^{2} / A\right)_{c r .}=35.5+0.4\left(Z_{f}-90\right)
$$

$Z_{f}$ is the charge of the fissioning nucleus. The critical fissility parameter $\left(Z^{2} / A\right)_{c r}$ for ${ }^{241} \mathrm{Am},{ }^{238} \mathrm{U}$, and ${ }^{237} \mathrm{~Np}$ are $37.5,36.3$, and 36.7 , respectively, while the average fissility parameter, $Z^{2} / A$, is 39.7 $(\mathrm{A}=227, \mathrm{Z}=95)$ for ${ }^{241} \mathrm{Am}, 37.3(\mathrm{~A}=227, \mathrm{Z}=92)$ for ${ }^{238} \mathrm{U}$, and $38.7(\mathrm{~A}=223, \mathrm{Z}=93)$ for ${ }^{237} \mathrm{~Np}$. The smallest difference between $Z^{2} / A$ and $\left(Z^{2} / A\right)_{c r}$ is found for ${ }^{238} \mathrm{U}$ and it could explain the larger contribution of asymmetric fission in the mass distribution for this nucleus. 

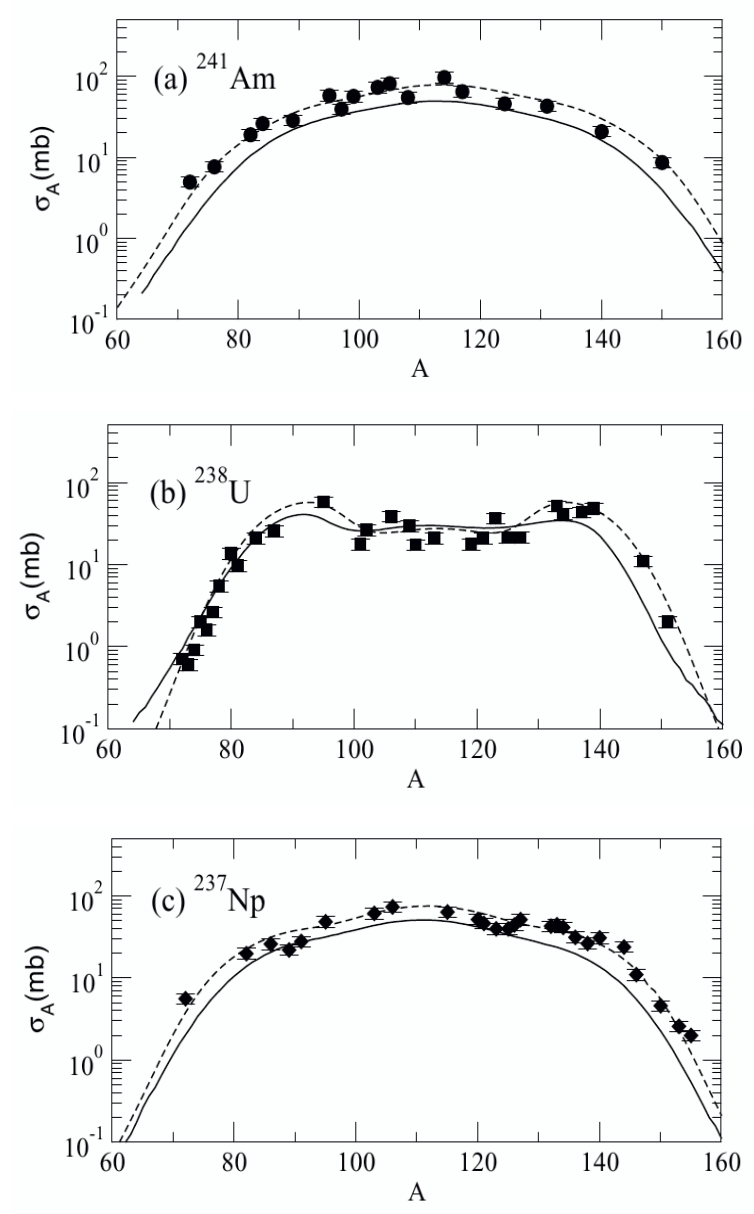

Figure 1. Mass distributions of fission fragments induced by 660 $\mathrm{MeV}$ protons on a) ${ }^{241} \mathrm{Am}$, b) ${ }^{238} \mathrm{U}$ and c) ${ }^{237} \mathrm{~Np}$ targets. The solid symbols are the experimental cross sections. The solid black line corresponds to calculation by CRISP and the dotted line is the result of a minimum $\chi$-square fitting over the data using Eq. (3).
The mass distributions of fragments for photon-induced reactions at $50 \mathrm{MeV}$ end-point energy are shown in Figure 2. A very good agreement is found at $50 \mathrm{MeV}$ end-point energy for both targets. A well parametrized multimodal approach can indeed reproduce the shell efects that lead to an asymmetric contribution at low energy. For higher energy, though, the symmetric contribution is greater and is consistently described by a classical liquid-drop model. Nevertheless, the multimodal-based calculation also takes this channel into account. The mass distributions of fragments for photon-induced reactions at $3500 \mathrm{MeV}$ end-point energy are shown in Figure 3. The agreement is not as good as for the previous results, particularly for what concerns the shift to the lighter fragments which is yet more pronounced for ${ }^{232} \mathrm{Th}$. This is due to excessive evaporation from the fragments and, although the model used for evaporation/competition is not perfectly ajusted for all possible targets and energies, the approximation of Eq. (6) already leaves the fragments with more available excitation energy than it should. A larger shift to the left can be observed in the region of heavier fragments but this should be expected in any case, since a partition of energy weighted by each fragment's mass is an accurate way of weighing.

The well-known fact that with high excitation energy there is an increase of symmetric fission, fact that is attested to in the experimental results at $3500 \mathrm{MeV}$ end-point energy, is also observed in the calculation by CRISP up to a certain point and with better success for ${ }^{232} \mathrm{Th}$. Another interesting aspect to point out is the prevalence of the symmetric contribution for ${ }^{232} \mathrm{Th}$ compared to ${ }^{238} \mathrm{U}$ in either case of the end-point energy. The Figure 4 may offer an explanation.

There is a larger number of lower mass fissioning systems in the case of ${ }^{232} \mathrm{Th}$ for both endpoint energies, besides the fact of being a lighter target. It is well-known that ${ }^{232} \mathrm{Th}$ has a lower fissility which leads, then, to a longer sequence of evaporation before fission. It is also of commom knowledge that neutrons comprise the majority of the emissions from which one concludes that more 

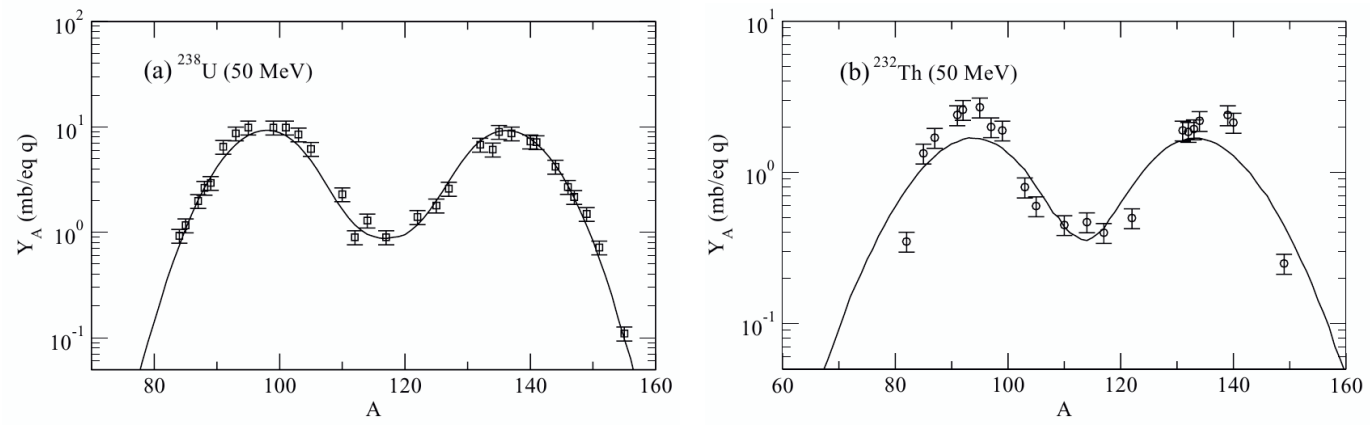

Figure 2. Mass distributions (yield) at $50 \mathrm{MeV}$ end-point energy for a) ${ }^{238} \mathrm{U}$ and b) ${ }^{232} \mathrm{Th}$. The solid line is the CRISP calculation.
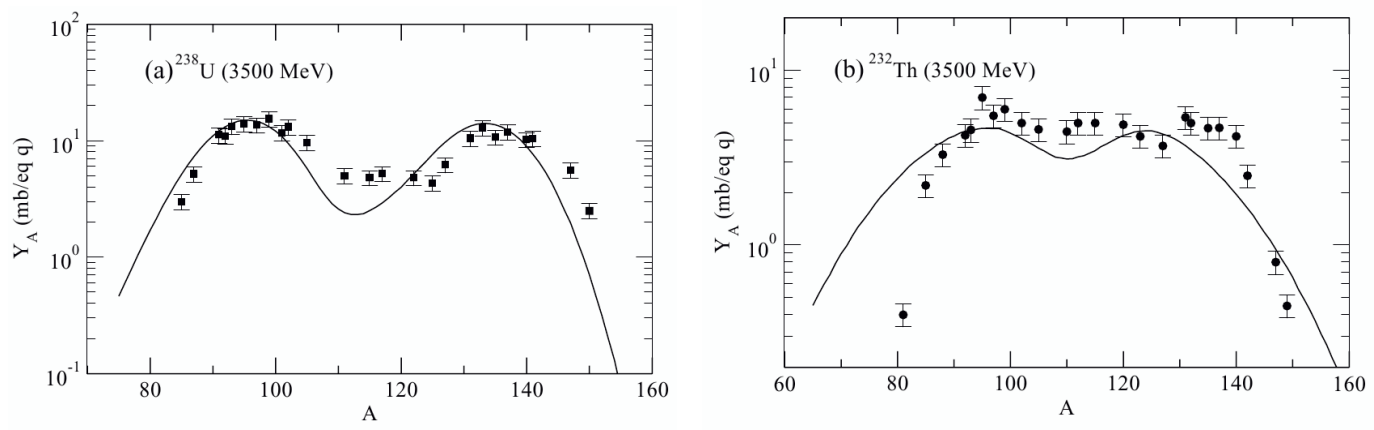

Figure 3. Mass distributions (yield) at $3500 \mathrm{MeV}$ end-point energy for a) ${ }^{238} \mathrm{U}$ and b) ${ }^{232} \mathrm{Th}$. The solid line is the CRISP calculation.

steps of evaporation give rise to more fissioning systems with high fissility parameter compared to ${ }^{238}$ U. According to Eq. (8), this would mean a larger contribution of the symmetric channel for ${ }^{232} \mathrm{Th}$.

\section{Conclusions}

We show that the CRISP code can give a reliable description of the fission dynamics for the reactions studied here. In fact, the mass distributions for fission fragments are correctly described by considering three fission modes, one symmetric and two asymmetric, for all targets studied. The evaporation of fission fragments is also considered, and we found that this mechanism is relevant for the description of the final fragment masses. In addition, the fissioning nucleus mass distributions can be a important source of knowledge in the task of explaining the relative contributions of symmetric and asymmetric channels, besides the increase or decrease of the excitation energy.

\section{Acknoledgments}

G.K. is grateful to Fundação de Amparo à Pesquisa do Estado de São Paulo (FAPESP) 2011/00314-0, and to the International Centre for Theoretical Physics (ICTP) under the Associate Grant Scheme. 

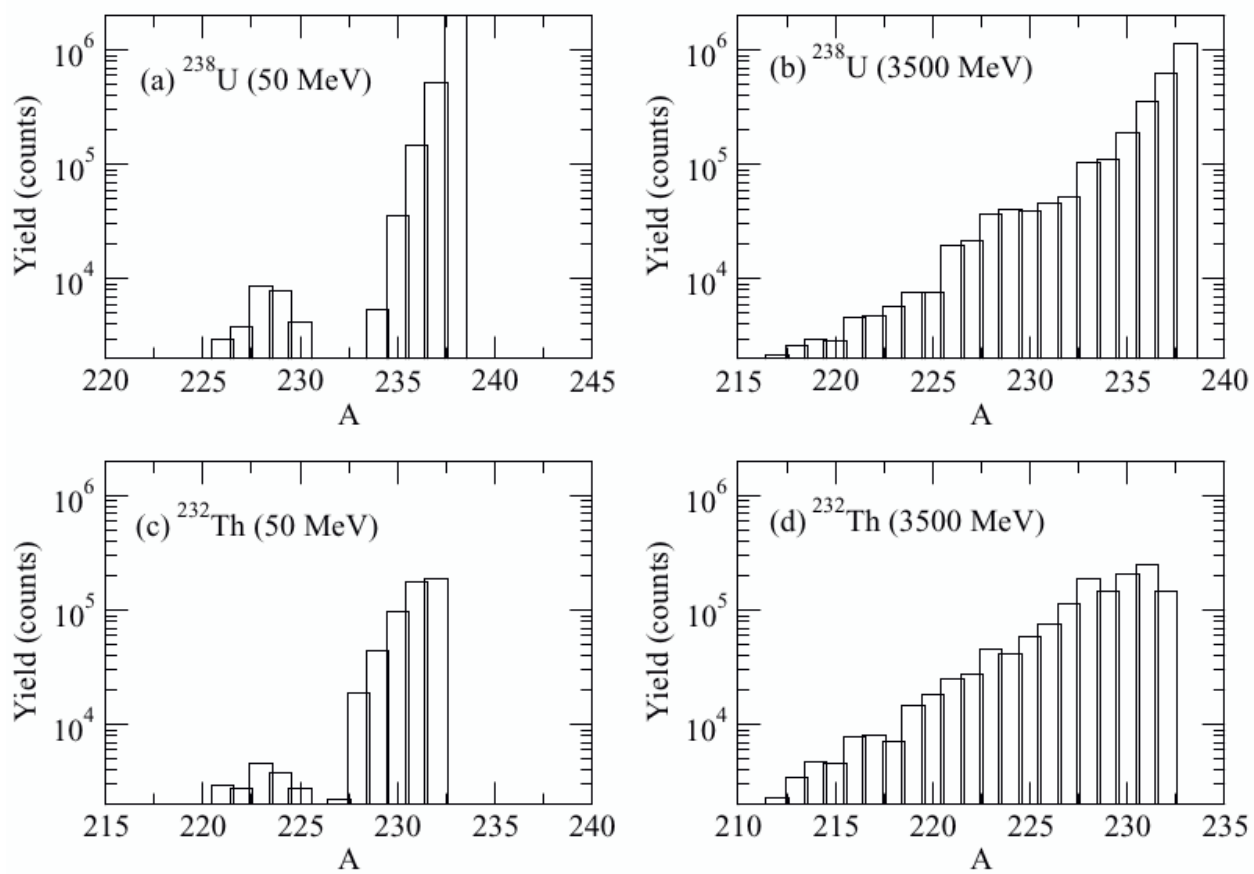

Figure 4. Mass distributions of the fissioning nucleus for ${ }^{238} \mathrm{U}, \mathrm{a}$ ) and b), and for ${ }^{232} \mathrm{Th}, \mathrm{c}$ ) and d).

A.D. acknowledge partial support from CNPq under grant 305639/2010-2 and FAPESP under grant 2010/16641-7. E.A. acknowledge support from FAPESP under grant 2012/13337-0.

\section{References}

[1] N. Bianchi et al., Phys. Lett. B 299, 219 (1993).

[2] J. C. Sanabria et al., Phys. Rev. C 61, 034604 (2000).

[3] C. Cetina et al., Phys. Rev. Lett. 84, 5740 (2000).

[4] G. S. Karapetyan, A. R. Balabekyan, N. A. Demekhina, and J. Adam, Phys. At. Nucl. 72, 911 (2009).

[5] A. R. Balabekyan, G. S. Karapetyan, N. A. Demekhina et al., Phys. At. Nucl. 73, 1814 (2010).

[6] N. A. Demekhina and G. S. Karapetyan, Phys. At. Nucl. 71, 27 (2008).

[7] N. A. Demekhina and G. S. Karapetyan, Phys. At. Nucl. 73, 24 (2010).

[8] A. Deppman, S. B. Duarte, G. Silva et al., J. Phys. G: Nucl. Part. Phys. 30, 1991 (2004).

[9] T. Kodama, S. B. Duarte, K. C. Chung, and R. A. M. S. Nazareth, Phys. Rev. Lett. 49, 536 (1982).

[10] M. Gonçalves, S. dePina, D. A. Lima et al., Phys. Lett. B 406, 1 (1997).

[11] A. Deppman, O. A. P. Tavares, S. B. Duarte et al., Phys. Rev. Lett. 87, 182701 (2001).

[12] A. Deppman, O. A. P. Tavares, S. B. Duarte et al., Comp. Phys. Comm. 145, 385 (2002).

[13] A. Deppman, O. A. P. Tavares, S. B. Duarte, J. D. T. Arruda-Neto, M. Gonçalves, V. P. Likhachev, and E. C. deOliveira, Phys. Rev. C 66, 067601 (2002). 
[14] A. Deppman, G. Silva, S. Anefalos et al., Phys. Rev. C 73, 064607 (2006).

[15] I. Gonzalez, C. Barbero, A. Deppman et al., J. Phys. G: Part. Nucl. 38, 115105 (2011).

[16] S. A. Pereira et al., Nucl. Sci. Eng. 159, 102 (2008).

[17] S. Anefalos, A. Deppman, G. Silva et al., Braz. J. Phys. 35, 912 (2005).

[18] S. T. Mongelli, J. R. Maiorino, S. Anefalos et al., Braz. J. Phys. 35, 894 (2005).

[19] V. F. Weisskopf and D. H. Ewing, Phys. Rev. 57, 472 (1940).

[20] U. Brosa, S. Grossman, and A. Muller, Z. Naturforsch. A 41, 1341 (1986).

[21] H. Kudo, M. Maruyama, M. Tanikawa, et al., Phys. Rev. C 57, 178 (1998).

[22] M. C. Duijvestijn, A. J. Koning, et al., Phys. Rev. C 59, 776 (1999).

[23] C. Chung and J. J. Hogan, Phys. Rev. C 25, 899 (1982). 